\title{
Contribution of meals and snacks to dietary intakes by Irish preschool children (1-4 years)
}

\author{
A.P. Nugent ${ }^{1,2}$, D.M. Martyn ${ }^{2}$, M.J. Gibney², J. Walton ${ }^{3,4}$, A. Flynn ${ }^{3}$, and B.A. McNulty2, \\ ${ }^{1}$ Institute for Global Food Security, School of Biological Sciences, Queens University Belfast, BT9 5HN, ${ }^{2}$ UCD Institute of Food and \\ Health, University College Dublin, Rep. of Ireland, ${ }^{3}$ School of Food and Nutritional Sciences, University College Cork, Rep. of Ireland, \\ ${ }^{4}$ Dept. Biological Sciences, Cork Institute of Technology, Rep. of Ireland
}

\section{Background:}

Globally, equivocal population guidance exists as to whether snack foods and snacking should form part of usual dietary intake patterns ${ }^{1,2}$. Further difficulties arise as there is no scientific consensus as to what constitutes a snack, a meal or an eating occasion ${ }^{1,2}$. Due to high energy and nutrient requirements relative to their body size, it is possible that young children may be more likely to consume foods and/or drinks between meals or to snack. The aim of this analyses was to characterize dietary intakes at meals and snacks in Irish 1-4 year olds.

\section{Methods:}

Analyses was based on the nationally representative, Irish National Preschool Nutrition Survey (NPNS) (2010-2011) which used a 4-d weighed food diary to collect detailed food and beverage intake data (www.iuna.net). Dietary intake data was analysed using WISP ${ }^{\odot}$, which is based on McCance and Widdowson's The Composition of Foods, Sixth $E_{\text {Edition }}$. For the present analysis, all eating occasions (EO) of less than OKJ and supplements were excluded with meal-types defined in the food diary by the recorder. All meal types were aggregated as 'breakfast', 'lunch', 'dinner', 'snacks' and 'beverages'. Included in 'beverages' were milks, formulae, juices and other juice drinks consumed as drinks. $\mathrm{PASW}{ }^{\odot} \mathrm{v} 20$ was used to calculate mean daily intake (MDI) of nutrients and the percentage at each EO.

\begin{tabular}{|c|c|c|c|c|c|c|c|c|c|c|}
\hline & \multicolumn{2}{|c|}{$\begin{array}{c}\text { Breakfast } \\
\text { (n499) }\end{array}$} & \multicolumn{2}{|c|}{$\begin{array}{l}\text { Lunch } \\
\text { (n500) }\end{array}$} & \multicolumn{2}{|c|}{$\begin{array}{l}\text { Dinner } \\
\text { (n496) }\end{array}$} & \multicolumn{2}{|c|}{$\begin{array}{l}\text { Snacks } \\
\text { (n494) }\end{array}$} & \multicolumn{2}{|c|}{$\begin{array}{l}\text { Beverages } \\
\text { (n293) }\end{array}$} \\
\hline & Mean & SD & Mean & SD & Mean & SD & Mean & SD & Mean & SD \\
\hline Energy (MJ/d) & $1.06^{a}$ & 0.36 & $1.12^{a b}$ & 0.48 & $1.13^{b}$ & 0.45 & $1.23^{b}$ & 0.70 & $0.53^{c}$ & 0.49 \\
\hline Energy (MJ/100g) & $0.48^{a}$ & 0.12 & $0.55^{b}$ & 0.17 & $0.52^{b}$ & 0.14 & $0.55^{b}$ & 0.30 & $0.25^{\mathrm{c}}$ & 0.07 \\
\hline Protein (\%TE) & $13.8^{\mathrm{a}}$ & 2.8 & $17.8^{\mathrm{b}}$ & 5.3 & $19.5^{\mathrm{c}}$ & 5.8 & $10.0^{d}$ & 3.3 & $15.2^{e}$ & 7.3 \\
\hline Fat (\%TE) & $26.5^{\mathrm{a}}$ & 10.3 & $34.2^{b}$ & 9.8 & $35.3^{b}$ & 9.5 & $29.6^{c}$ & 10 & $36.7^{b}$ & 22.1 \\
\hline Saturated fat (\%TE) & $13.7^{\mathrm{a}}$ & 7.4 & $14.8^{\mathrm{b}}$ & 5.6 & $14.2 \mathrm{ab}$ & 5.2 & $14.1 \mathrm{ab}$ & 5.7 & $20.1^{c}$ & 15.5 \\
\hline Carbohydrate (\%TE) & $55.2^{\mathrm{a}}$ & 9.3 & $45.8^{\mathrm{b}}$ & 10.5 & $43.6^{c}$ & 10.5 & $58.0^{d}$ & 10.8 & $42.4^{\mathrm{c}}$ & 19.7 \\
\hline Sugars (\%TE) & $25.6^{a}$ & 9.4 & $19.2^{b}$ & 9.9 & $16.4^{c}$ & 9.1 & $38.7^{d}$ & 13.7 & $39.3^{d}$ & 19.0 \\
\hline NMES (\%TE) & $14.0^{a}$ & 9.7 & $13.4^{\mathrm{a}}$ & 8.1 & $11.3^{\mathrm{a}}$ & 7.3 & $23.5^{b}$ & 12.1 & $16.0^{\mathrm{a}}$ & 27.7 \\
\hline Lactose (\%TE) & $8.9^{a}$ & 4.7 & $2.7^{b}$ & 2.8 & $3.1^{b}$ & 3.4 & $5.0^{\mathrm{c}}$ & 4.7 & $23.1^{d}$ & 12.1 \\
\hline Fibre (g/10MJ) & $26.2^{a}$ & 11.5 & $26.4^{a}$ & 15.1 & $27.1^{a}$ & 12.6 & $27.0^{a}$ & 17.1 & $12.1^{b}$ & 22.5 \\
\hline Sodium (mg/10MJ) & $1893^{a}$ & 811 & $3373^{b}$ & 1516 & $3121^{b}$ & 1445 & $1820^{a}$ & 939 & $2326^{c}$ & 4419 \\
\hline
\end{tabular}

\%TE: percentage contribution to total energy; intake values are compared between the meal types using one way ANOVA, controlled for age and gender, with Bonferroni post hoc tests; abc different superscripts denote significance between meal types at $P<0.05$. NMES non milk extrinsic sugars

\section{Results:}

On average, children consumed each of 3 main meals, 2 snacks and 1 beverage EO daily. Breakfast was the most micronutrient dense meal for nutrients such as iron, calcium, folate and thiamin, perhaps reflecting high user rate and contribution to energy intakes at breakfast for foods such as non-sugar coated ready to eat break breakfast cereals (89\% users, $31 \%$ energy contribution), milk, cheese and yogurts (96\% users, $30 \%$ energy contribution) and breads and rolls $(60 \%$ users, $9 \%$ energy contribution).

\section{Conclusion:}

All EO types made significant contributions to energy and nutrient intake by Irish preschool children. Future work should consider how meal and snacking patterns may change with age into older childhood and beyond.

\section{References: \\ 1. Potter M, Vlassopoulos A \& Lehmann U (2018) Adv Nutr, 9(2),86-98. 3. Hess JM, Jonnalagadda SS, Slavin JL (2016) Adv Nutr, 7(3),466-75 Murakami B \& Livingstone MBE (2016) J Acad Nutr Diet 116(7), \\ Food Standa \\ Soon Standards Agency (2002) Royal Society of Chemistry.}

Acknowledgement:

Funded by the Irish Department of Agriculture, Food and the Marine under the project 'National Children's Food

Consumption Survey II' (15/F/673)
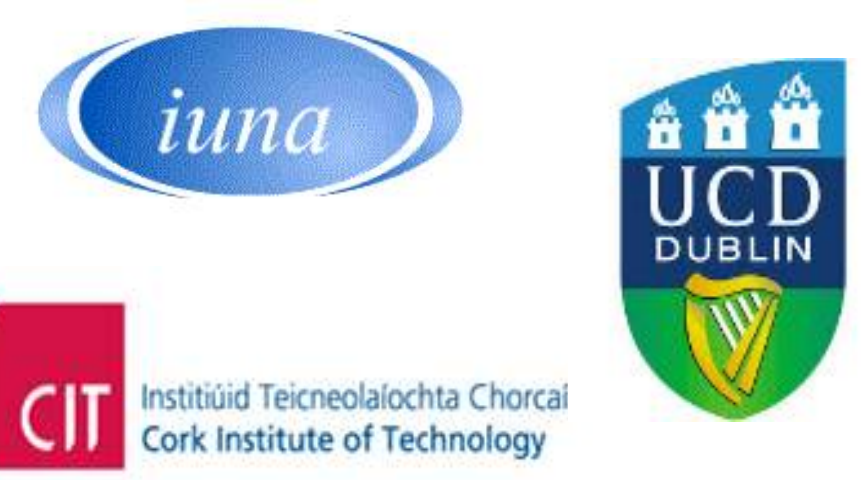
BELFAST 